Review

\title{
Capacitive Mixing for Harvesting the Free Energy of Solutions at Different Concentrations
}

\section{Raúl A. Rica ${ }^{1,2}$, Roberto Ziano ${ }^{1}$, Domenico Salerno ${ }^{1}$, Francesco Mantegazza ${ }^{1}$, René van Roij ${ }^{3}$ and Doriano Brogioli ${ }^{1, *}$}

${ }^{1}$ Dipartimento di Scienze della Salute, Università di Milano-Bicocca, via Cadore 48, Monza (MB), Italy; E-Mails: rul@ugr.es (R.A.R.); roberto.ziano@unimib.it (R.Z.); domenico.salerno@unimib.it (D.S.); francesco.mantegazza@unimib.it (F.M.)

${ }^{2}$ ICFO-The Institute of Photonic Sciences, Av. Carl Friedrich Gauss, num. 3, Castelldefels, Spain

${ }^{3}$ Institute for Theoretical Physics, Utrecht University, Leuvenlaan 4, 3584 CE Utrecht, The Netherlands; E-Mail: r.vanroij@uu.nl

* Author to whom correspondence should be addressed; E-Mail: dbrogioli@gmail.com.

Received: 18 March 2013; in revised form: 2 April 2013 / Accepted: 14 April 2013 /

Published: 17 April 2013

\begin{abstract}
An enormous dissipation of the order of $2 \mathrm{~kJ} / \mathrm{L}$ takes place during the natural mixing process of fresh river water entering the salty sea. "Capacitive mixing" is a promising technique to efficiently harvest this energy in an environmentally clean and sustainable fashion. This method has its roots in the ability to store a very large amount of electric charge inside supercapacitor or battery electrodes dipped in a saline solution. Three different schemes have been studied so far, namely, Capacitive Double Layer Expansion (CDLE), Capacitive Donnan Potential (CDP) and Mixing Entropy Battery (MEB), respectively based on the variation upon salinity change of the electric double layer capacity, on the Donnan membrane potential, and on the electrochemical energy of intercalated ions.
\end{abstract}

Keywords: free energy; water salinity difference; capacitive mixing

\section{Introduction}

The first reference to energy production from water salinity difference dates back to 1954, when Pattle reported this resource together with a proof-of-concept [1]: "When a volume $V$ of a pure solvent mixes 
irreversibly with a much larger volume of a solution the osmotic pressure of which is $P$, the free energy lost is equal to $P V$. The osmotic pressure of sea-water is about 20 atmospheres, so that when a river mixes with the sea, free energy equal to that obtainable from a waterfall 680 feet. high is lost. There thus exists an untapped source of power which has (so far as I know) been unmentioned in the literature". The energy thus dissipated comes from the free diffusion experienced by the ions from the salt (sea) water to the fresh (river) water, which increases the ionic entropy and accordingly decreases the Gibbs or Helmholtz energy of the final solution.

The amount of energy dissipated due to the mixing of two solutions with different salt concentrations has been evaluated several times in the context of the colloquially called "Blue Energy", or more properly referred to as salinity difference energy [2-6], so we only pose here a simplified analysis of this quantity. Consider two volumes $V_{\text {fresh }}$ and $V_{\text {salt }}$ of two solutions with molar salt concentrations $c_{\text {fresh }}$ and $c_{\text {salt }}$, respectively, of a monovalent binary electrolyte (such as $\mathrm{NaCl}$ ). After mixing, the final state is a volume $V_{\text {tot }}=V_{\text {fresh }}+V_{\text {salt }}$ of brackish water of concentration $c_{\text {brackish }}=\left(c_{\text {fresh }} V_{\text {fresh }}+c_{\text {salt }} V_{\text {salt }}\right) / V_{\text {tot }}$. The Gibbs free energy of mixing per unit volume of the final (brackish) solution is given by the difference between the final and the initial state:

$$
-\Delta G_{\text {mix }} \simeq 2 R T\left[c_{\text {brackish }} \ln c_{\text {brackish }}-\phi c_{\text {fresh }} \ln c_{\text {fresh }}-(1-\phi) c_{\text {salt }} \ln c_{\text {salt }}\right]
$$

where $R$ is the gas constant, $T$ is absolute temperature and $\phi=V_{\text {fresh }} / V_{\text {tot }}$ is the volume fraction of fresh water. Equation 1 sets the thermodynamic upper limit for obtainable work in any controlled mixing process, which is only achieved in the case of a reversible cycle with no global entropy production.

In order to harvest the free energy, the mixing has to be performed in a controlled way, for which many techniques have been developed since the 1950s, but none of them has yet fulfilled the commercial requirements. A suitable technique should provide an amount of energy that is considerably larger than that consumed in the pre-treatment. Along this point, a trade-off can be found between the pretreatment energy and the duration of the membranes, as pre-treatments consuming more energy are expected to lead to longer membrane life. Considerations like the cost per unit power of the plant (also expressed as payback time) and the cost per unit produced energy including maintenance and replacement of consumable parts (e.g., membranes) are also important. Finally, the efficiency of energy conversion, i.e., the ratio between the produced power and the quantity of water is also an issue, as water itself, particularly the fresh stream, has a cost. Classical approaches are mainly based on the use of membranes, and some recent reviews focus on their advances [4,7-11] and therefore they are not addressed in the present work.

Three novel methods have been proposed, associated with the ability to selectively store and release anions or cations into ion-adsorbing electrodes (supercapacitors) [12-15] from the bulk solution. Altogether, they are now called the "Capacitive Mixing" (CAPMIX) techniques, as the storage of ions takes advantage of the capacitances or pseudo-capacitances of several types of electrodes [16,17]. CAPMIX techniques include "Capacitive Double Layer Expansion" (CDLE) [18], "Capacitive Donnan Potential" (CDP) [19] and "Mixing Entropy Battery" (MEB) [20]. The ion selectivity is reached in CDLE by charging the electrodes by means of an external power supply; CDP uses permselective membranes, which are selective to the transport of ions through them, as they allow the passage of only one type of ions (cations or anions); MEB has two different electrodes that specifically react with only one species of 
ion. All of them can be operated performing a four-step cycle during which the solution is alternatively exchanged between the fresh water and the saltwater streams.

As we show below, thermodynamic arguments serve to directly connect CAPMIX with existing deionization techniques that consume energy to remove ions from a saltwater stream, adsorbing them from it into the electrodes to later release them, producing brine as a waste. Generally accepted as the reverse processes of the CAPMIX techniques are the "Capacitive Deionization" (CDI) [21,22], reverse of CDLE; "Membrane Capacitive Deionization" (MCDI) [23,24], reverse of CDP; and the "Desalination Battery" (DB) [25], reverse of MEB.

In this work we first review the thermodynamic basis of the CAPMIX techniques (Section 2), and then we identify the common features in order to give a general picture of this new strategy (Section 3). Finally, we discuss the recent advances of each technique (Section 4) and we make an overview of the required future steps to arrive to a mature and competitive technology (Section 5).

\section{The CAPMIX Family}

In the broadest scheme, the CAPMIX cycle consists in charging a pair of "generalized electrodes" or "accumulators" to reach a given potential when submerged in one solution, and in discharging them at a lower potential while submerged in the other solution [17,26,27]. We use the terms generalized electrodes or accumulators in order to include any kind of electrode or assembly that is able to temporarily store charge, either capacitively, with membranes, in redox reactions, or through ion intercalation. Therefore, as we discuss below, the charging mechanism depends on the used electrodes. The key point in the CAPMIX cycle is thus the sequential exchange of the bulk solution in which the electrodes are submerged, leading to changes of their capacitance (or pseudo-capacitance in the case of $\mathrm{MEB}$ ) and, therefore, of the cell potential. Although not mandatory, it is common to perform the solution exchange in open circuit configuration, thus keeping the stored charge constant.

The capacitance $C$ is related to the stored charge $Q$ and the cell voltage $\varphi$ through:

$$
\varphi=\frac{Q}{C}
$$

From this relation, it is clear that a decrease in the capacitance at constant charge (open circuit) will return an increase in the cell potential, and therefore an increase of the stored electrochemical energy $E$, which for the case that $\mathrm{C}$ is independent of $\mathrm{Q}$ and $\varphi$, reads:

$$
E=\frac{1}{2} Q \cdot \varphi=\frac{1}{2} \frac{Q^{2}}{C}
$$

Three different types of electrodes have been tested to extract energy from salinity differences by means of a general CAPMIX cycle [16,17]. Whether the cell is charged in the salt- or in the freshwater depends on the properties of the employed electrodes and on the origin of their selectivity for charge storage.

The first proposed CAPMIX technique was CDLE, which exploits the dependence of the capacitance of "Electric Double Layer" (EDL) supercapacitors [12] on salt concentration. CDP technique appeared soon after CDLE, and it includes permselective membranes to benefit from the Donnan potential that develops across them. Finally, MEB does not store charge capacitively, but pseudo-capacitively as 
chemical energy inside the electrode bulk crystal structure, and makes use of the dependence of the Nernst potential on salt concentration.

\subsection{Capacitive Double Layer Expansion}

The pioneering work of Brogioli [18] involved a pair of activated carbon porous electrodes to perform the cycle represented in Figure 1 and is composed by the four following phases:

- Phase A: The cell, filled with saltwater, is charged from an initial potential $\varphi_{\mathrm{D}}$ to a final potential $\varphi$ charge through an external load. In this phase energy is stored into the cell.

- Phase B: In open circuit, i.e., at constant charge, the solution in the cell is exchanged with freshwater. The voltage increases to $\varphi_{\mathrm{B}}>\varphi_{\text {charge, }}$, and the amount of energy stored in the cell also increases, due to the decrease of the capacitance.

- Phase C: The cell is discharged to the potential $\varphi_{\text {charge }}$ through an external load. In this phase, energy is extracted from the cell.

- Phase D: Again in open circuit, the solution in the cell is exchanged with saltwater. The voltage decreases to $\varphi_{\mathrm{D}}$ due to the increase of the capacitance.

Figure 1. Representation of the four-step CAPMIX cycle on the potential $(\varphi)$ versus charge $(Q)$ graph as published in [18] where the area represents the extracted energy. The power produced was about $7 \mathrm{~mW}$ per square meter of electrode. Copyright (2009) by The American Physical Society.

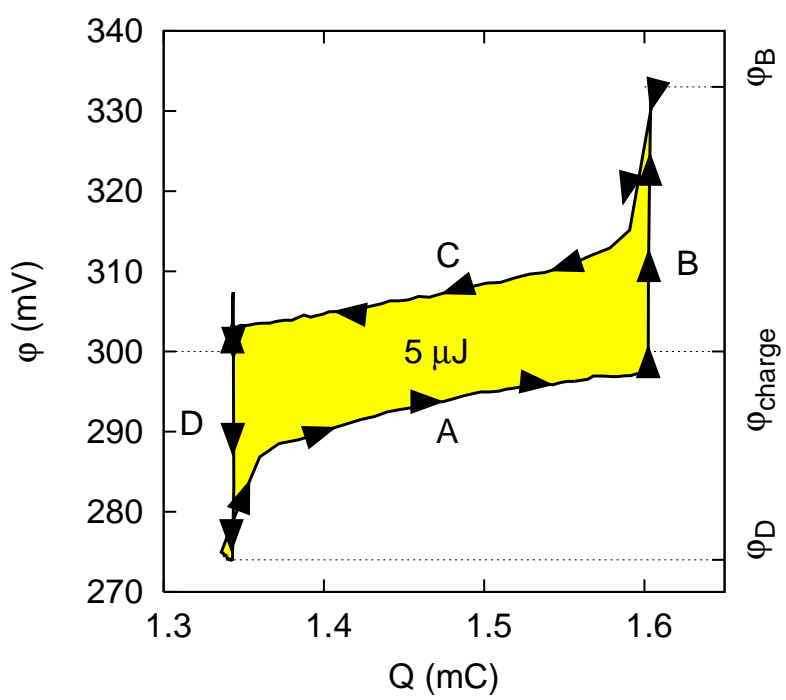

The cycle represented in Figure 1 is able to extract an amount of work $W=-\oint \varphi d Q$ because the capacitance of the cell depends on the bulk salt concentration through the properties of the EDL. This is the ionic distribution established close to a charged surface in contact with an ionic solution, due to the competition between the electrostatic forces attracting counter-ions and repelling co-ions and the diffusive forces trying to equilibrate the concentration of every species. A simplified picture considers both types of ions distributed according to Boltzmann statistics, allowing us to define the characteristic 
length of this distribution as the Debye length $\lambda_{\mathrm{D}}$ [28]. In the case of a monovalent electrolyte, the expression of the $\lambda_{\mathrm{D}}$ reads:

$$
\lambda_{\mathrm{D}}=\frac{1}{\sqrt{8 \pi \lambda_{\mathrm{B}} c}}
$$

$c$ being the bulk ions concentration (either $c_{\text {fresh }}$ or $c_{\text {salt }}$ ) expressed in number of ions per unit volume, and $\lambda_{\mathrm{B}}=e^{2} / 4 \pi \varepsilon_{r} \varepsilon_{0} k_{\mathrm{B}} T$ the Bjerrum length, where $\varepsilon_{r}$ is the relative electric permittivity of the liquid, $\varepsilon_{0}$ the electric permittivity of vacuum, $e$ the proton charge and $k_{\mathrm{B}}$ is the Boltzmann constant. At room temperature, $\lambda_{\mathrm{B}} \simeq 7.1 \AA$ and the Debye length takes values $\lambda_{\mathrm{D}} \in[10,0.4] \mathrm{nm}$ for $c \in[1,600] \mathrm{mM}$, the salt concentration of river and seawater, respectively. From this equation, it is clear that the EDL will be thicker the lower the salt concentration, and vice versa. This dependence of $\lambda_{\mathrm{D}}$ on bulk salt concentration is the origin of the term "double layer expansion". The voltage drop in the diffuse part of the EDL, $\Delta \varphi_{\mathrm{DL}}$, can be described by the Gouy-Chapman surface charge-voltage relationship [28]:

$$
\Delta \varphi_{\mathrm{DL}}(\sigma, c)=2 \frac{k_{\mathrm{B}} T}{e} \sinh ^{-1}\left(2 \pi \lambda_{\mathrm{B}} \lambda_{\mathrm{D}} \frac{\sigma}{e}\right)
$$

where $\sigma$ is the total charge per unit surface available for charge adsorption on the electrode. From this equation, we see that the voltage drop in the diffuse part of the double layer increases when $c$ is decreased at constant $\sigma$, and that the specific (per unit surface) capacitance of the EDL $C_{\text {surf }}=\sigma / \Delta \varphi_{\text {DL }}$ decreases with $c$.

\subsection{Capacitive Donnan Potential}

Sales et al. [19] proposed a similar technique to CDLE that can be operated without external power supply, by adding ion-exchange membranes to the CAPMIX cell, thus taking advantage of the Donnan potentials that develop across such membranes. Ideally, ion-exchange membranes allow the passage of only one type of ion through them (either the cation or the anion), and set up a net current if an anion-exchange membrane (AEM) and a cation-exchange membrane (CEM) are placed in front of the two electrodes, as shown in Figure 2a. The driving force for ionic current in CDP is the Donnan potential that is established between two reservoirs with different salinity separated by an ion-exchange membrane:

$$
\Delta \varphi_{\text {Donnan }}\left(c_{\text {fresh }}, c_{\text {salt }}\right)=\alpha \frac{k_{\mathrm{B}} T}{e} \ln \left(\frac{c_{\text {fresh }}}{c_{\text {salt }}}\right)
$$

where $\alpha$ is the average selectivity of the membrane, which can be also expressed as the difference in the transport numbers across the membrane $\alpha=\left(t_{+}-t_{-}\right)$[26,29], where $t_{+}$and $t_{-}$(with $t_{+}+t_{-}=1$ ) are the transport numbers of cations and anions, respectively [30]. Therefore, a cell voltage increment is observed when the solution in the spacer channel is changed due to the presence of such Donnan potentials between the two sides of each electrode. This voltage difference induces ionic currents that store charge into the electrodes by the formation of EDLs at the electrode/solution interface [19]. Furthermore, the charge induced in the electrode-side of the EDL forces an electronic current in the external circuit. Alternating fresh- and saltwater in the spacer channel produces a continuous power production, as shown in Figure $2 b$. 
Figure 2. (a) Schematic representation of a CDP CAPMIX cell, with ion-exchange membranes in front of each electrode, separated by a spacer channel for the alternate flow of fresh- and saltwater; (b) Measured cell voltage and power production in a two-phase CDP CAPMIX cell. Dark blue: saltwater; Light blue: freshwater. Adapted with permission from [19]. Copyright 2010 American Chemical Society.
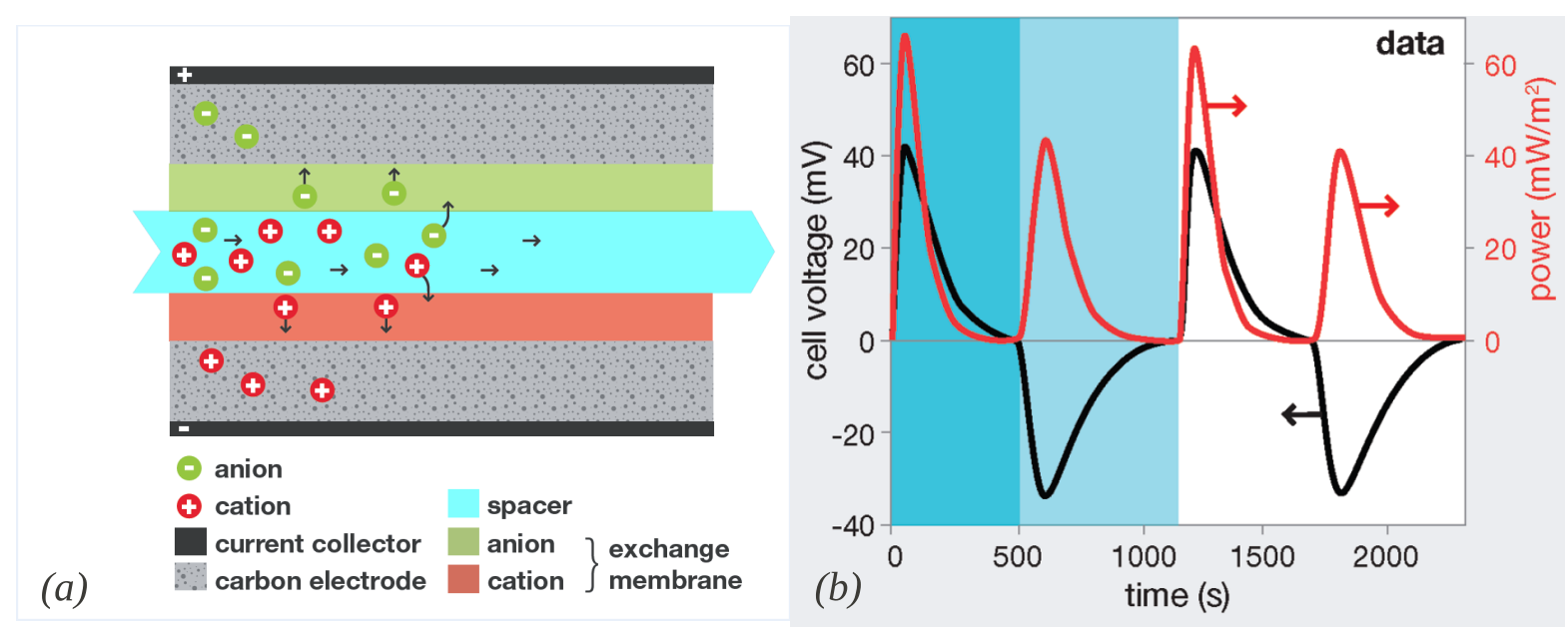

Therefore, the CDP CAPMIX cycle proposed by Sales et al. [19] consists of only two phases, namely when the cell is submerged in fresh- and saltwater, without open circuit steps, allowing a continuous (although not constant) power production. Such a cycle produced peaks of about $60 \mathrm{~mW}$ per square meter of electrode and membranes.

\subsection{Mixing Entropy Battery}

Typical problems associated with the use of activated carbon in supercapacitor electrodes include the sensitivity to impurities and dissolved oxygen, which are partially responsible for the observed leakage phenomena [18,31] or the fouling of nanopores. To overcome these problems La Mantia et al. [20] proposed a novel electrochemical cell where the ionic charge storage takes place inside the electrode material's bulk crystal structure. The "Mixing Entropy Battery" tested by La Mantia et al. takes advantage of the specific interactions of a $\mathrm{Na}_{2} \mathrm{Mn}_{5} \mathrm{O}_{10}$ electrode with $\mathrm{Na}^{+}$ions and an $\mathrm{Ag} / \mathrm{AgCl}$ reference electrode with $\mathrm{Cl}^{-}$ions. In this technique, the equilibrium potential of these two chemical reactions depends on the salt concentration, thus allowing the extraction of energy from the controlled mixing of fresh- and saltwater.

As can be seen in Figure 3, the equilibrium potential is higher in saltwater than in freshwater, and therefore the CAPMIX MEB cycle is different from the CDLE and CDP ones, since in this case the cell is charged in freshwater (by removing $\mathrm{Cl}^{-}$and $\mathrm{Na}^{+}$ions from the $\mathrm{AgCl}$ and $\mathrm{Na}_{2} \mathrm{Mn}_{5} \mathrm{O}_{10}$ electrodes, respectively), while it is discharged in saltwater (by capturing $\mathrm{Cl}^{-}$and $\mathrm{Na}^{+}$ions into the $\mathrm{AgCl}$ and $\mathrm{Na}_{2} \mathrm{Mn}_{5} \mathrm{O}_{10}$ electrodes, respectively) at a higher equilibrium potential, thus producing a net amount of energy. Indeed, La Mantia et al. report a power production of $105 \mathrm{~mW} / \mathrm{m}^{2}$, of the same order of those obtained for CDLE and CDP [17,32,33]. 
Figure 3. (a) Schematic representation of the working principle behind a complete cycle of the mixing entropy battery, showing how energy extraction can be accomplished: step 1, charge in river water; step 2, exchange to seawater; step 3, discharge in seawater; step 4, exchange to river water; (b) Evolution during the four-step cycle of the individual potentials of the two electrodes of a MEB experiment measured with respect to a $\mathrm{Ag} / \mathrm{AgCl}$ reference electrode; (c) Measured cycle of battery cell voltage $(\Delta E) v s$. charge $(q)$ in a mixing entropy battery, demonstrating the obtainable energy. Reprinted with permission from [20]. Copyright 2011 American Chemical Society.
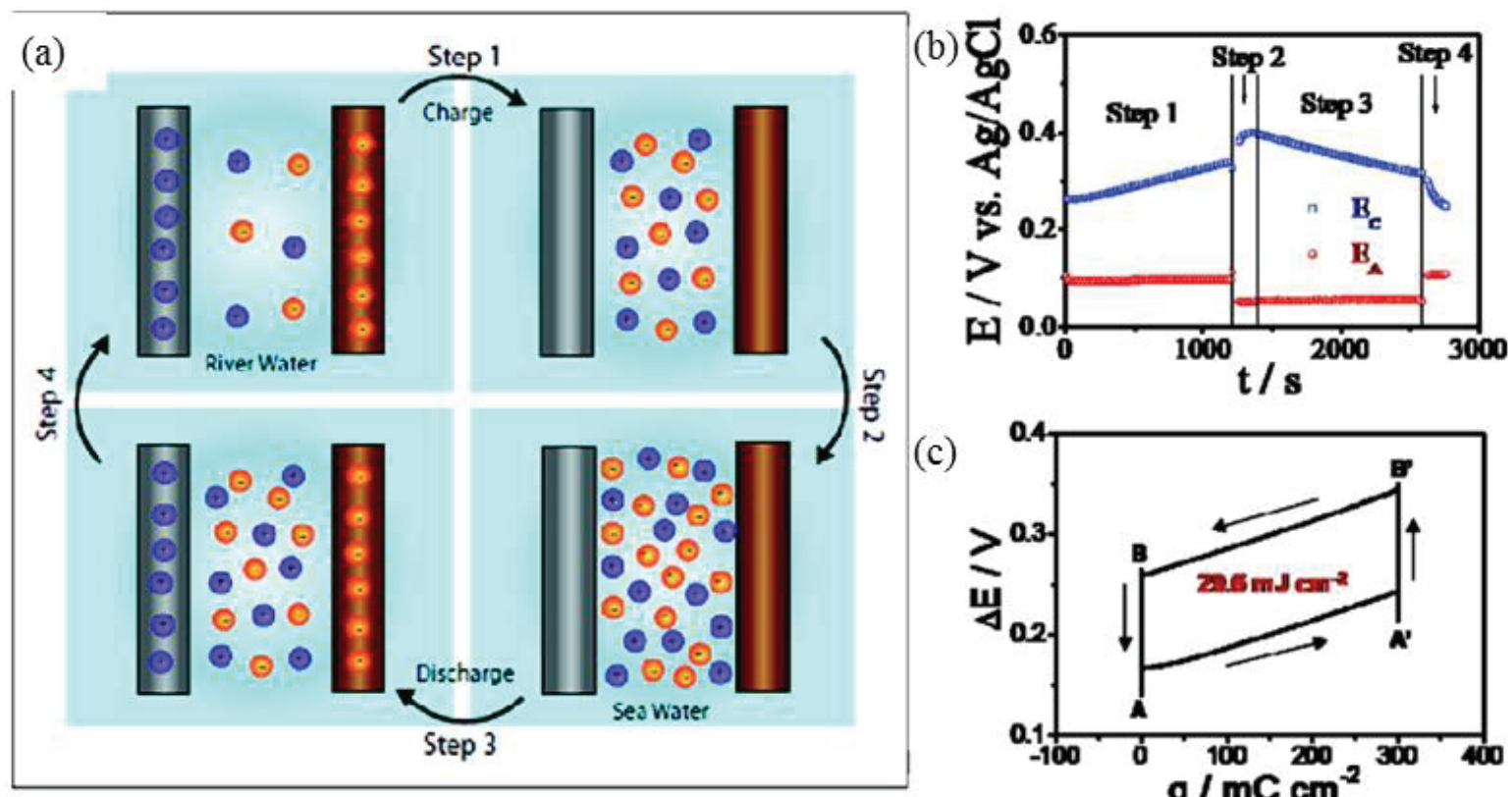

(c)

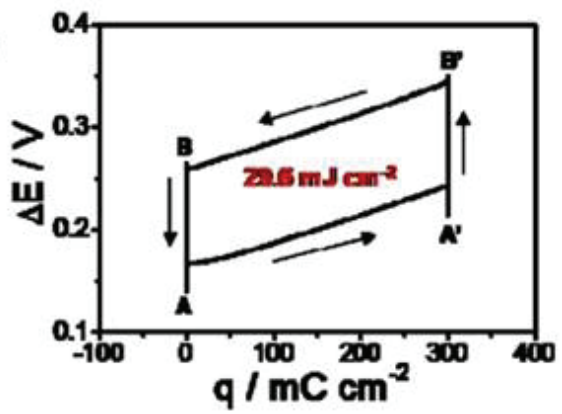

\section{Common Features of the CAPMIX Techniques}

\subsection{Thermodynamics of "Blue Engines" and CAPMIX Cycles}

Boon and Van Roij [5], van Roij [27] and we [26] have shown that thermodynamic arguments can be very informative of the CAPMIX process regardless of the details of the particular technique. Along this view, Boon and van Roij propose the study of the performance of a "Blue engine" composed by a pair of electrodes submerged in an 1:1 electrolyte, calculating the Helmholtz potential $F(T, V, N, Q)$ of a half-cell of volume $V$ at temperature $T$ containing a single electrode, assuming symmetric behavior of the other one. The system has one electrode with total charge $Q$ (so the other electrode has a charge $-Q$ ). In the whole cell, there are $N$ counterions and $N$ co-ions, so that the system electrodes-electrolyte is electroneutral. Keeping the temperature $T$ and the geometric properties of the electrode (surface area, porosity, volume, pore curvature, etc.) fixed, we can simplify notation writing $F(N, Q)$, such that the differential of the Helmholtz potential is:

$$
d F=\mu d N+\varphi d Q
$$


where the ionic chemical potential $\mu=\left(\frac{\partial F}{\partial N}\right)_{Q}$ and the electrostatic potential of the electrode $\varphi=\left(\frac{\partial F}{\partial Q}\right)_{N}$ have been defined. The work performed by the system in a cycle is $W=-\oint \varphi d Q$. If the cycle is performed reversibly, $\oint d F=0$, and hence the work also equals

$$
W=\oint \mu d N
$$

i.e., a reversible CAPMIX cycle must involve exchange of ions between the electrode and its medium [27].

Interestingly, we can demonstrate that Equation 8 implies that a reversible CAPMIX cycle performed with the CDLE technique that is able to produce work $(W>0)$ involves intrinsically non-linear screening phenomena, in the same way as CDI is a nonlinear phenomenon on the stored charge $[21,22]$. In fact, together with charge adsorption into the EDL, salt adsorption will also take place in the charging process. The origin of capacitive salt adsorption is not trivial, and resides in the mechanism of charge storage into the electric double layers (EDL) that form at the interface between the carbon electrodes and the solution [26,34]. The charge in the EDL stems not only from an increase of the concentration of counter-ions but also from a decrease of the concentration of co-ions, the latter contribution becoming smaller than the former for increasing charge (second order in $\sigma$ ), resulting in a net ion concentration increase, i.e., a salt storage into the EDLs in the couple of electrodes [26,34]. The salt adsorption per unit surface in the Gouy-Chapman model is given by [34]:

$$
\Gamma_{\mathrm{DL}}(\sigma, c)=\sqrt{\left(\frac{\sigma}{e}\right)^{2}+\left(\frac{1}{2 \pi \lambda_{\mathrm{B}} \lambda_{\mathrm{D}}}\right)^{2}}-\frac{1}{2 \pi \lambda_{\mathrm{B}} \lambda_{\mathrm{D}}}= \begin{cases}\frac{\sigma^{2}}{2 e \sigma^{*}}, & \text { if }|\sigma| \ll \sigma^{*} \\ \frac{|\sigma|}{e}, & \text { if }|\sigma| \gg \sigma^{*}\end{cases}
$$

with the crossover surface charge $\sigma^{*}=e / 2 \pi \lambda_{\mathrm{B}} \lambda_{\mathrm{D}}$. The crossover behavior from $\Gamma \propto \sigma^{2}$ at low $\sigma$ to $\Gamma=$ $|\sigma| / e$ at high $\sigma$ signifies a qualitative change from the linear screening regime, where the double layers exchange co-ions for counter ions keeping the total ion concentration fixed (such that $\Gamma$ is negligible), to the nonlinear screening regime where counterion condensation takes place, and a net number of ions is adsorbed into the EDLs.

The total number of ions in the system (the whole cell composed by the two electrodes) $N_{\text {tot }}$ can be written in terms of the adsorption and the bulk concentration $c$ as:

$$
N_{\text {tot }}=2 c V+\Gamma A=2 N
$$

where $A$ is the area of one electrode. For $N$ to change during a reversible cycle in which work is produced, the salt adsorption $\Gamma$ must be different from zero. Therefore, as $\Gamma$ is a second order (nonlinear) screening phenomenon [26,34], a reversible CDLE CAPMIX cycle is in turn essentially non-linear. However, an irreversible CAPMIX cycle can be operated to produce a net amount of work in the linear regime. In fact, Boon and van Roij were able to identify the CAPMIX cycle in Figure 1 with the Stirling cycle (which, in fact, can be used to extract energy even at low $\sigma$ ) and to propose the most efficient alternative based on an analogy with the (reversible) Carnot cycle [5,27], where salt adsorption and desorption upon charging in saltwater and discharging in freshwater, respectively, play a fundamental role. The Carnot-like cycles include a desalination step of the saltwater due to ion adsorption during electrode charging at constant $N$ (such that $c$ reduces according to Equation 10), and a resalination 
step due to ion desorption during electrode discharging at constant $N$ (such that $c$ increases according to Equation 10). Due to these desalination and resalination steps in the cycle (which are the analogues of the adiabatic cooling and heating steps in the expansions and compression steps of the thermal Carnot cycle, respectively), no irreversible mixing takes place in the cycle, such that it is reversible in ideal conditions.

In CDP, the thermodynamic reversibility of a cycle that is able to generate energy (see Equation 8) requires ideal membranes, i.e., $\alpha=1$, so that irreversible mixing does not take place. If the membranes are indeed ideal, the required salt adsorption from the spacer channel into the generalized electrode (the assembly electrode + membrane, usually referred to as membrane-electrode assembly, MEA) is always present, as any charging process implies the transport of only one type of ions (the counterion of the membrane), and the number of ions inside the electrode always increases upon charging. Therefore, contrary to CDLE, CDP can be operated in the linear regime of stored charge in a reversible manner.

Like in the case of CDP, the MEB cycle can be performed reversibly, as irreversible mixing of the two solutions can be avoided. The condition of increasing the number of ions $2 N$ in the system required by reversibility (see Equation 8) is also fulfilled, i.e., there is always adsorption of a net number of ions into the electrodes. This can be understood by observing that a couple of positive and negative ions react and are adsorbed into the electrodes for each two electrons that are moved from the positive to the negative electrode, and thus always contribute to ion adsorption [26]:

$$
\Gamma_{\mathrm{MEB}}=\frac{|\sigma|}{e}
$$

Moreover, in a recent paper van Roij identified several Maxwell-like relations by taking second derivatives of the Legendre transformations of the state function $F(N, Q)$ [27,35]. These thermodynamic identities must hold regardless of the nature, composition, geometry, etc., of the electrode and the electrolyte. Of particular interest is the Maxwell relation that stems from the grand potential $\Omega(\mu, Q)=$ $F-\mu N$, such that $d \Omega=-N d \mu+\varphi d Q$. By considering $\partial^{2} \Omega / \partial \mu \partial Q$ one easily derives the Maxwell relation

$$
\left(\frac{\partial \varphi}{\partial \mu}\right)_{Q}=-\left(\frac{\partial N}{\partial Q}\right)_{\mu}
$$

If one now realizes that the electrolyte bulk concentration $c$ does not change at constant $\mu$, one can write the right hand side of this equation as $(\partial \Gamma / \partial \sigma)_{c}$, where $\Gamma$ is the adsorption (per unit area) of salt onto an electrode and $\sigma$ the total charge (per unit area) of the electrode. We can thus rewrite this Maxwell relation for homogeneous electrodes as

$$
\left(\frac{\partial \varphi}{\partial \mu}\right)_{\sigma}=-\left(\frac{\partial \Gamma}{\partial \sigma}\right)_{c}
$$

Interestingly, an alternative derivation of this Maxwell relation and its experimental verification have been proposed [26]. The expression presented in [26] reads (in the current notation)

$$
\frac{c}{2 R T}\left(\frac{\partial \varphi}{\partial c}\right)_{\sigma}=-\left(\frac{\partial \Gamma}{\partial \sigma}\right)_{c}
$$

and follows directly from Equation 13 by inserting the ideal-gas relation $\mu=2 R T \ln c$. The minus sign that is not present in the expression of [26] is due to a different trivial sign convention of the potential. 
Equation 14 connects the rise of the electrode potential upon a salinity change at constant electrode charge to the salt adsorption upon rising the charge at constant salinity. In other words, this relation poses a direct link between the Brogioli-type CAPMIX cycle on the one hand and the salt adsorption upon charging on the other, i.e., it provides an explicit link between the performances of CAPMIX and the corresponding deionization techniques (the aforementioned CDI, MCDI and DB). From this relation, we conclude that technical improvements in the performance of these desalination techniques should also be applicable to improvements on CAPMIX, and vice versa [26].

\subsection{Ions Transport and Adsorption in a CAPMIX Cell}

The dynamics of all the CAPMIX techniques are strongly influenced by the transport of ions through all the components of a CAPMIX cell, including flow channels, membranes and porous electrodes. Experiments have revealed a complex evolution of the cell potential during the CAPMIX cycle, especially in the open circuit steps. A proper description of the dynamics thus requires the understanding of the transport of ions across ion-selective membranes (where present) and inside porous media, probably at quite different size scales, together with the (capacitive or pseudo-capacitive) storage of ions in the electrodes, i.e., adsorption and desorption.

A good approximation to such understanding can be achieved through the volume averaged model for porous structures developed by Biesheuvel and Bazant [36], although more complex treatments are also available [37-42]. The model in [36] describes how the electro-diffusion of ions inside a porous matrix is affected by adsorption and desorption of ions into and from the electrodes, respectively. The adsorption mechanisms can be capacitive (CDLE and CDP), pseudo-capacitive (MEB) or chemical (when faradaic reactions are present $[37,41,43]$ ), and lead to perturbations of the local salt concentration (salination and desalination) inside the electro-neutral macropores (with a size of the order of $1 \mu \mathrm{m}$ ) that serve as pathways for the transport of ions, see Figure 4. Although these works only consider the transport in one dimension (perpendicular to the flow in the spacer channel, see Figure 4), they can (and should) be extended to other dimensions (especially along the flow-channel, where non-uniform charge and salt adsorption is expected $[44,45])$.

Such analysis has already been used by us to analyze the dynamics of the CDLE CAPMIX cycle [44,45], finding excellent agreement between the model and experiments [44]. The basis of this treatment is the formulation of one dimensional diffusion and Ohm equations in porous media, extended to account for charge $j_{\text {charge }}$ and salt $j_{\text {salt }}$ adsorption into the electrodes [36]. In dimensionless form (see the original papers for more details $[36,37,44,45])$, the diffusion and Ohm equations are, respectively:

$$
\begin{gathered}
\frac{\partial \tilde{i}(\tilde{x}, \tilde{t})}{\partial \tilde{x}}=\frac{\partial}{\partial \tilde{x}}\left(-\tilde{c}(\tilde{x}, \tilde{t}) \frac{\partial \tilde{\phi}(\tilde{x}, \tilde{t})}{\partial \tilde{x}}\right)=-\frac{\gamma}{p_{\mathrm{mA}}} \tilde{j}_{\text {charge }}(\tilde{x}, \tilde{t}) \\
\frac{\partial \tilde{c}(\tilde{x}, \tilde{t})}{\partial \tilde{t}}=\tilde{D} \frac{\partial^{2} \tilde{c}(\tilde{x}, \tilde{t})}{\partial \tilde{x}^{2}}-\frac{\gamma}{p_{\mathrm{mA}}} \tilde{j}_{\text {salt }}(\tilde{x}, \tilde{t})
\end{gathered}
$$

where $\tilde{x}$ and $\tilde{t}$ are dimensionless position (perpendicular to the flow in the channel, see Figure 4) and time; $\tilde{i}(\tilde{x}, \tilde{t})$ is a specific, dimensionless current; $\tilde{D}$ is a normalized diffusion coefficient; $\tilde{c}(\tilde{x}, \tilde{t})$ and $\tilde{\phi}(\tilde{x}, \tilde{t})$ are the (dimensionless) local salt concentration and electric potential; $\gamma$ and $p_{\mathrm{mA}}$ are a measure 
of the space available for adsorption and macroporosity, respectively, both defined per unit volume of electrode; and $\tilde{j}_{\text {charge }}(\tilde{x}, \tilde{t})$ and $\tilde{j}_{\text {salt }}(\tilde{x}, \tilde{t})$ are the (dimensionless) rates of charge and salt adsorption into the electrodes, respectively. $\tilde{j}_{\text {charge }}(\tilde{x}, \tilde{t})$ and $\tilde{j}_{\text {salt }}(\tilde{x}, \tilde{t})$ cancel out of the porous matrix, and therefore Equations 15 and 16 reduce to the classic Ohm law $\left(\tilde{i}(\tilde{x}, \tilde{t})=-\tilde{c}(\tilde{x}, \tilde{t}) \frac{\partial \tilde{\phi}(\tilde{x}, \tilde{t})}{\partial \tilde{x}}\right)$ and pure diffusion $\left(\frac{\partial \tilde{c}(\tilde{x}, \tilde{t})}{\partial \tilde{t}}=\tilde{D} \frac{\left.\partial^{2} \tilde{c} \tilde{x} \tilde{t}\right)}{\partial \tilde{x}^{2}}\right)$ in the spacer channel.

Figure 4. Schematic representation of a generic CAPMIX cell (other geometries and configurations are possible), composed by two parallel plate electrode assemblies (made of a porous material with or without a ion-exchange membrane in front of it) separated by a spacer channel where fresh- and saltwater flow alternatively. The external power supply can also be absent (CDP). Without losing generality, the enlarged area represents the vicinity of the interface between the spacer channel (where ions are transported by electro-diffusion and convection) and the cathode (covered with a cation-exchange membrane in the case of CDP), along which ions are transported by electro-diffusion and are adsorbed and desorbed into EDLs or the crystal structure of the electrodes. Arrows in the enlarged area represent ions transport and adsorption/desorption in the electrodes.

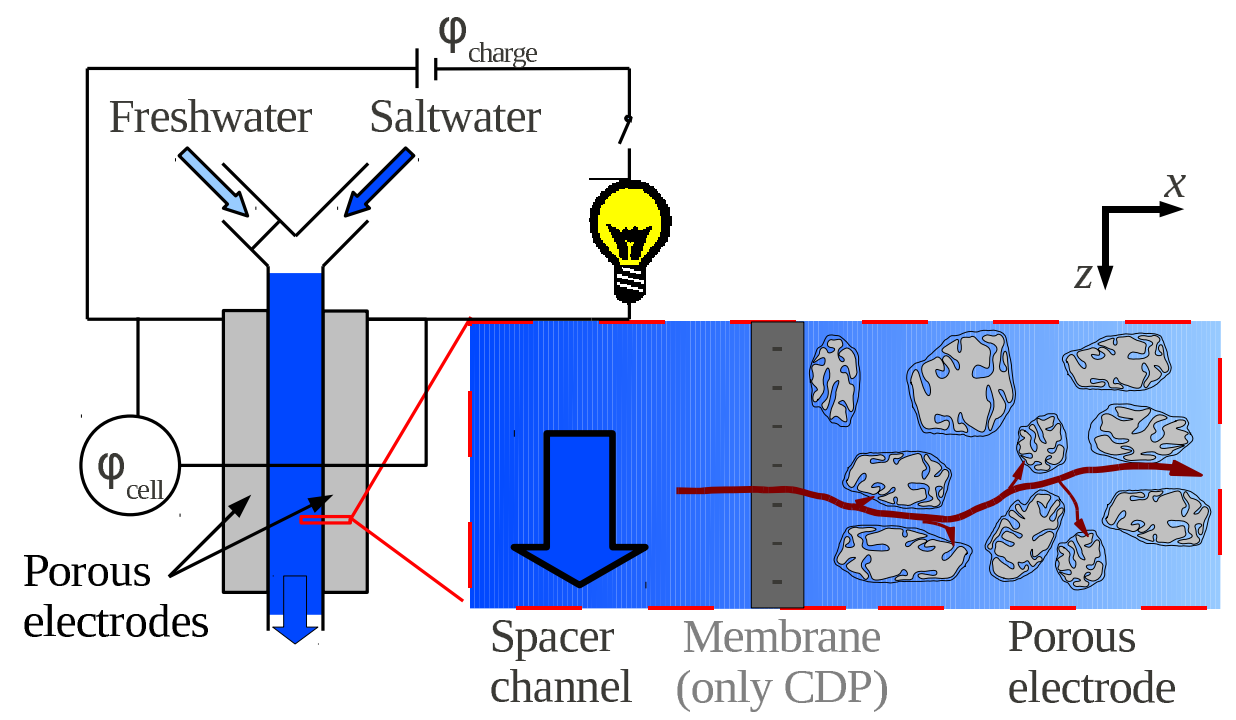

The general formulation given by Equations 15 and 16 with appropriate boundary conditions at the spacer/(membrane)/porous matrix interfaces and at the back of the electrodes, where a current collector is present $[36,44]$, is applicable to all the CAPMIX techniques and also to batteries or fuel cells $[36,46,47]$. Furthermore, it can be extended to more dimensions and other geometries, such as the wire-like electrodes recently proposed [48]. The dynamics of a CDP CAPMIX cycle with wire geometry has been recently studied considering the diffusion in the concentration-polarization layer close to an ion-selective membrane, without including the transport and adsorption of ions into the electrodes [49]. The authors of [49] obtain qualitative agreement between theory and experiments, and although their results could also be of interest in CDLE, its applicability is more restricted than the model proposed here for both CDP and CDLE. In fact, we discussed in [44,45] that Equations 15 and 16 predict non-uniform charge and salt adsorption in the electrodes, and that this non-uniform adsorption effectively influence 
the dynamics of the CDLE CAPMIX cell, especially for large voltages, small macroporosity and large space available for ions adsorption. Non-uniform charge and salt adsorption are also expected to take place in CDP and MEB, and therefore the transport along the porous electrodes and these adsorption mechanisms also have to be considered to obtain a full description of these processes.

\subsection{Key Parameters}

For the evaluation of the feasibility of the application of a given type of electrode, the most important parameters that need to be characterized are the voltage rise due to the salinity change and the overvoltage, i.e., the part of the measured cell voltage due to currents in the internal resistances. With the membrane-based electrodes used in CDP and the battery electrodes used in MEB, the voltage rise is given by Nernst law, i.e., it is around $80 \mathrm{mV}$ per electrode in sea/river water. On the other hand, it is limited by the properties of the EDLs for the activated carbon electrodes used in CDLE.

In the case of CDLE, the overvoltage is mainly due to the gap between the electrodes $[44,45]$. For $\mathrm{CDP}$, the main contribution to the cell overvoltage comes from the resistance of the membranes and is a well studied problem in electrochemical cells and batteries. For this reason, many results obtained in the field of electrochemistry can be translated into results for CAPMIX.

Therefore, the research that is being done in order to optimize the performance of CAPMIX has its focus on the maximization of the voltage rise, effectively extracting the energy from the controlled mixing of the two solutions, and minimizing the overvoltage, i.e., the energy lost in the internal resistance of a CAPMIX cell.

The operation of the CAPMIX cell is also of singular importance for the maximization of the extracted power. Relevant investigations along this line of research include the optimization of cycle timing, i.e., the duration of each step of the cycle $[44,45,49]$; the minimization of the time needed to exchange the solutions [33] and the avoidance of the mixing of the two solutions in the flow channel $[31,33,44]$.

\section{Recent Advances}

Besides thermodynamic arguments, many physical and engineering issues have to be addressed and solved before any of the CAPMIX techniques becomes commercially competitive for renewable energy harvesting from salinity differences. These issues include the selection and optimum fabrication of the most suitable materials, appropriate cycle and cell designs, operation conditions, fouling prevention, cleaning and maintenance methods, choosing suitable locations, etc. Below we review the recent advances in their development.

\section{1. $C D L E$}

After the proof-of-concept provided by Brogioli [18], new lab scale prototypes have been built and tested with the Brogioli cycle [17,31,44], considerably improving the power output. However, they have also revealed two important problems to be faced, which together limit the maximum power that can be extracted. The main one is the presence of a strong charge leakage in the CDLE cell [31] (and also in CDP [19,32]), which is mainly apparent in the open circuit steps, when the solutions are exchanged. 
The second one deals with the long transients observed in the switching steps $[18,31,44]$, when the cell voltage evolves with bulk salt concentration following a quite complex dynamics.

Although mandatory to overcome these difficulties, a full understanding of all the involved adsorption mechanisms is still lacking, but some important steps forward have already been taken. The first theoretical analysis of the CDLE technique considered the aforementioned Gouy-Chapman model for EDLs, which assumes that they are locally flat [18,31]. However, suitable electrodes are made of porous materials composed of activated carbon particles, commonly used as supercapacitors thanks to their huge specific surface and, therefore, capacitance $[12,15,50]$. These materials have typical pore sizes of the order of some nanometers, size range where the EDL cannot be considered locally flat. Moreover, the Gouy-Chapman is a mean-field theory of point-like ions, returning unrealistically large ion concentration and capacitances at large applied potentials [51,52]. Some improved descriptions have already been proposed. In [52], realistic predictions of the energy that can be extracted by solving the whole set of electrokinetic equations [50,53] with a cell model [54] are provided, allowing for partial overlap of EDLs and considering the finite size of ions. The authors of this work identify optimal performances for small pore sizes [52], in agreement with recent experiments showing larger capacitances and best CDI performances with nanopores [50,55,56]. Moreover, the authors in [52] also identify the optimal "working" or external potential ( $\varphi_{\text {charge }}$ in the present notation) to be around $250 \mathrm{mV}$. Boon and Van Roij [5] use a density functional including EDLs overlap and finite ion size to calculate ionic adsorption and desorption in porous electrodes, proposing modifications of the Brogioli CAPMIX cycle. Finally, strongly overlapping EDLs have also been considered, approximation valid in the limit of small pore size $[44,45]$.

The dynamics of CDLE has also been studied, considering the effect of ions transport inside the porous matrix and their adsorption/desorption into the EDLs. The electro-diffusion model developed by Biesheuvel and Bazant [36] has been successfully applied to simulate the CAPMIX cycle, providing a full description of the four-phase Brogioli-cycle [44,45]. This study explains the complex dynamics of the CAPMIX cycle as mainly due to the strongly non-linear voltage-concentration dependence characteristic of the EDL and on non-uniform charge adsorption in the electrode, making the length and the thickness of the employed electrodes a very important parameter to be finely tuned. Moreover, the macroporosity, i.e., the micron-size pores inside the electrodes that serve as pathways for ion transport [36], is shown to be very important on the dynamic response of an electrode. Large macroporosity facilitates transport, thus decreasing characteristic response times, but also decreases the specific capacitance of the electrode. Therefore, a compromise between these two effects is to be found [45].

Finally, the issue of charge leakage in CDLE experiments has been systematically investigated, obtaining exciting results regarding the actual behavior of carbon electrodes [17]. Experiments have shown that carbon electrodes leak charge during CAPMIX cycles [17,31,32], avoiding an efficient energy extraction from salinity differences with the CDLE technique, as it is frequent that more power is leaked than can be extracted. The physical mechanism of charge leakage is not clear, and is attributed to specific interactions and chemical reactions at the solid/liquid interface. According to [17], the leakage works to shift the electrode potential toward a fixed value, the spontaneous potential of the electrode. This spontaneous potential depends not only on the properties of the material (chemical 
composition, crystal structure, surface treatment, pore size, etc.) but also on salt concentration. The breakthrough obtained in [17] consists in taking advantage of the different concentration dependence presented by some materials that have a similar spontaneous potential but opposite evolution upon salinity exchange, different from the expected from the double layer expansion mechanism, and allows to operate the Brogioli-type CAPMIX cycle without an external power supply. This means that the EDL super-capacitors employed in these experiments do not behave as ideally polarizable materials, but present pseudo-capacitances characteristic of battery electrodes [57,58], similarly to those used by La Mantia et al. [20] for MEB.

The strategy used by Brogioli et al. [17] to get rid of both the leakage and the external power supply consists in using two different electrodes with the same spontaneous voltage but opposite behavior in the CAPMIX cell, i.e., one electrode that increases its potential upon changing from salt- to freshwater and vice versa, and other that decreases its potential upon such exchange and vice versa. The proof-of-principle is illustrated in Figure 5, where a charged CAPMIX cell is subjected to alternative flows of salt- and freshwater (without discharging it). As we see, the two electrodes have a similar spontaneous voltage of about $220 \mathrm{mV}$ in salt water, while the voltage difference between them increases when we switch to freshwater. Adding charge/discharge steps to this two-phase cycle, energy can be extracted without a power supply and with almost no leakage [17]. In this work, Brogioli et al. [17] reached a power production of $50 \mathrm{~mW}$ per $\mathrm{m}^{2}$ of electrode without any optimization, an encouraging result and very promising in the pursue of a competitive technology.

Figure 5. Open circuit potential variation upon salinity change of the two electrodes of a CAPMIX cell made of two different activated carbon materials (MCC and A-PC-2) measured with respect to a reference electrode. The cycles were obtained without external charging, as reported in [17]. Copyright 2012 by The Royal Society of Chemistry.

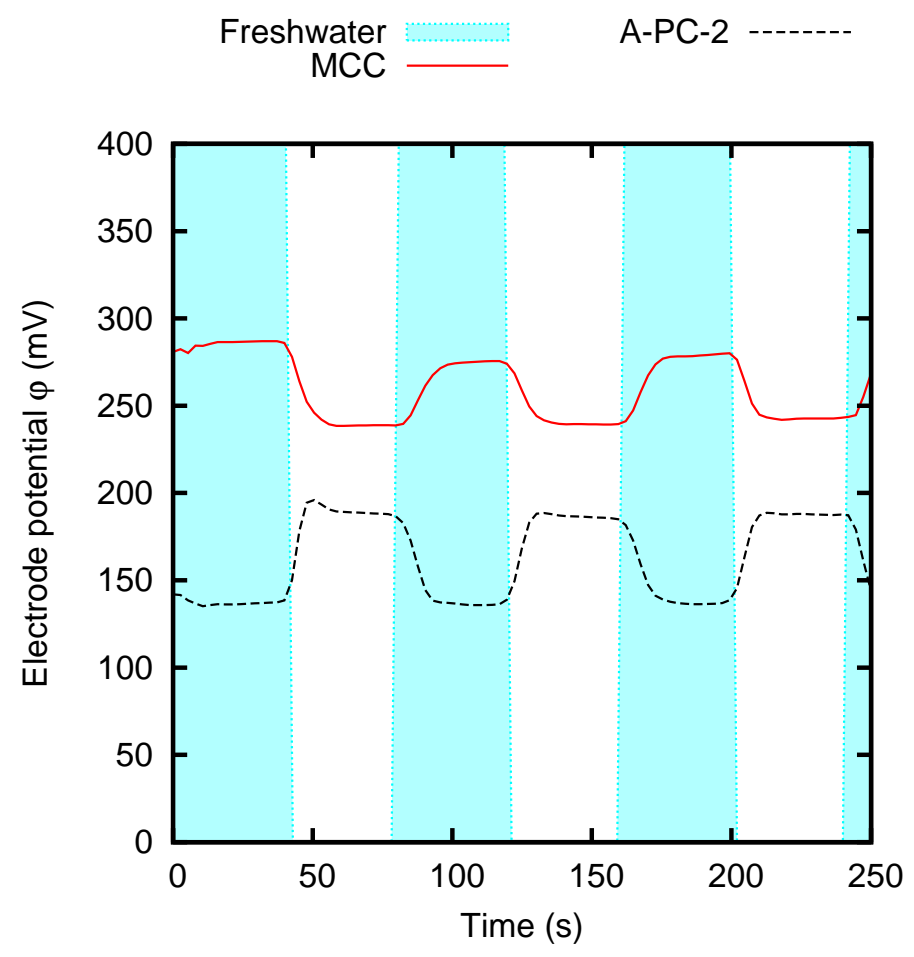




\section{2. $C D P$}

Several publications have already contributed to the advancement and development of CDP with very relevant results $[32,48,49,59]$. These studies include modifications of the CDP cycle proposed by Sales et al. [19] and of the geometry of electrodes and the cell itself, together with some theoretical descriptions of the process. They certainly constitute important improvements, but much work is still to be done, both from the technological and in the theoretical points of view.

In [32], it is shown that the energy extraction can be improved by increasing the stored charge with a forced current, summing up to the charge driven by the Donnan potential. Moreover, they analyze different operations for the discharge process, which is shown to be optimal if performed at low, constant current, due to better impedance matching. With these modifications, the authors reach a power production of about $200 \mathrm{~mW} / \mathrm{m}^{2}$, still subject to optimization. The phenomenological description provided in this work accounts for all the effects observed in experiments, but a full theoretical description is still lacking.

The main issues to be tackled in order to further increase the power production include the minimization of the internal resistance of the cell, thus minimizing ohmic losses; minimization or avoidance of leakages due to non-ideal behavior of electrodes [17,57] and membranes [60], responsible for voltage decreases during open circuit steps and irreversible mixing of the different solutions, respectively; and the minimization of switching time, which imposes a transient time for the stabilization of the cell voltage that significantly enlarges the duration of the CDP CAPMIX cycle, similarly to the case of CDLE.

Among these, the internal resistance was identified as the main hurdle in [32]. A good improvement in this direction has been recently achieved [48] using a different geometry for the CDP cell, found to be useful in CDI [61]. In these works, it is proposed to use wire-shaped electrodes, instead of the flowthrough parallel plate above described. Such geometry seems to strongly reduce the internal resistance between two wires as compared with that expected for two parallel plates, thus reducing the important ohmic losses observed in the discharging phase of the cycle, which is performed in freshwater. Moreover, Sales et al. [48] claim that wire-shaped electrodes would reduce the hydraulic resistance of the cell, further improving its performance.

As already said, the dynamics of the open circuit potential upon salinity exchange in the case of wire-shaped electrodes for CDP has been analyzed by studying the diffusion of salt in the thin, unstirred layer adjacent to the membrane surface in cylindrical geometry [49]. This theoretical analysis is satisfactorily compared with experiments on the transient behavior of the open circuit potential, finding a somewhat faster response of wire-shaped electrodes than planar ones.

Therefore, in the light of the recent improvements on CDP with wire-shaped electrodes, it seems that this strategy presents advantages with respect to the planar geometry. However, future research in this direction must evaluate if these advancements are enough to compensate the expected higher production costs of wires in comparison with planar electrodes. 


\section{Perspectives}

Naturally occurring salinity differences are not limited to delta areas. Brines are available in salt lakes (e.g., Dead Sea), coal-mine brines [62] produced by dissolving geological deposits. They can be artificially produced by means of salterns or as a by-product of desalination plants. Moreover, the brines can be used versus sea water, thus avoiding the freshwater consumption.

Salinity differences can also be produced by distillation. The required heat can be obtained by a renewable source (e.g., by means of a solar concentrator), or can be the waste heat from an industrial process, or part of a co-generation process. Also in this case, the technique contributes to the renewable and clean energy production.

Therefore, the development of CAPMIX cells cannot be limited to sodium chloride solutions with concentrations $500 \mathrm{mM}$ and around $20 \mathrm{mM}$, but must include other solutions. For example, $\mathrm{LiFePO}_{4}$ battery electrodes have been proposed to be used with lithium chloride solutions, which can be obtained by solar distillation [20]. Applications of reverse electrodialysis is currently under study [63], which has been recently combined with capacitive technology to reach improved performances [64]. It is likely that different salts will lead to different electrokinetic behaviors, and some particular combination of a CAPMIX technique with a particular solution could lead to an improved efficiency.

Maybe the most important open front in the development of CAPMIX deals with the use of novel kinds of electrodes, possibly combining different techniques in hybrid supercapacitors. In principle, any electrode used for energy storage can be potentially used in CAPMIX. A clear proof of this is the number of alternatives to the initial idea of purely capacitive storage that have appeared soon after the first CAPMIX paper by Brogioli.

In conclusion, we are optimistic with the thought that some of these recent techniques, or a suitable combination of them, will be found to be an efficient tool for the extraction of renewable energy from the entropy produced in the controlled mixing of solutions with different salt concentrations.

\section{Acknowledgements}

The research leading to these results received funding from the European Union Seventh Framework Programme (FP7/2007-2013) under agreement no. 256868. R.A.R. acknowledges support from Regione Lombardia (Accordo per lo sviluppo del capitale umano nel sistema universitario lombardo). F.M. and D.S. acknowledge support of Cariplo Foundation Materiali Avanzati -2011, Project 2011-0336.

\section{References}

1. Pattle, R.E. Production of electric power by mixing fresh and salt water in the hydroelectric pile. Nature 1954, doi:10.1038/174660a0.

2. Norman, R.S. Water salination: A source of energy. Science 1974, 186, 350-352.

3. Levenspiel, O.; de Vevers, N. The osmotic pump. Science 1974, 183, 157-160.

4. Labrecque, R. Exergy as a useful variable for quickly assessing the theoretical maximum power of salinity gradient energy systems. Entropy 2009, 11, 798-806. 
5. Boon, N.; van Roij, R. "Blue energy" from ion adsorption and electrode charging in sea and river water. Mol. Phys. 2011, 109, 1229-1241.

6. Yip, N.Y.; Elimelech, M. Thermodynamic and energy efficiency analysis of power generation from natural salinity gradients by pressure retarded osmosis. Environ. Sci. Technol. 2012, 46, 5230-5239.

7. Post, J.W.; Veerman, J.; Hamelers, H.V.M.; Euverink, G.J.W.; Metz, S.J.; Nymeijer, K.; Buisman, C.J.N. Salinity-gradient power: Evaluation of pressure-retarded osmosis and reverse electrodialysis. J. Membr. Sci. 2007, 288, 218-230.

8. Ramon, G.Z.; Feinberg, B.J.; Hoek, E.M.V. Membrane-based production of salinity-gradient power. Energy Environ. Sci. 2011, 4, 4423-4434.

9. Logan, B.; Elimelech, M. Membrane-based processes for sustainable power generation using water. Nature 2012, 488, 313-319.

10. Chung, T.S.; Li, X.; Ong, R.C.; Ge, Q.; Wang, H.; Han, G. Emerging forward osmosis (FO) technologies and challenges ahead for clean water and clean energy applications. Curr. Opin. Chem. Eng. 2012, 1, $246-257$.

11. Siria, A.; Poncharal, P.; Biance, A.L.; Fulcrand, R.; Blase, X.; Purcell, S.; Bocquet, L. Giant osmotic energy conversion measured in a single transmembrane boron nitride nanotube. Nature 2013, 494, 455-458.

12. Conway, B. Electrochemical Supercapacitors; Kluwer Academic, Plenum Publishers: London, UK, 1999.

13. Kötz, R.; Carlen, M. Principles and applications of electrochemical capacitors. Electrochim. Acta 2000, 45, 2483 - 2498.

14. Aricò, A.S.; Bruce, P.; Scrosati, B.; Tarascon, J.; van Schalkwijk, W. Nanostructured materials for advanced energy conversion and storage devices. Nat. Mater. 2005, 4, 366-377.

15. Simon, P.; Gogotsi, Y. Materials for electrochemical capacitors. Nat. Mater. 2008, 7, 845-854.

16. Bijmans, M.F.M.; Burheim, O.S.; Bryjak, M.; Delgado, A.; Hack, P.; Mantegazza, F.; Tennisson, S.; Hamelers, H.V.M. CAPMIX- Deploying capacitors for salt gradient power extraction. Energy Procedia 2012, 20, 108-115.

17. Brogioli, D.; Ziano, R.; Rica, R.A.; Salerno, D.; Kozynchenko, O.P.; Hamelers, H.V.; Mantegazza, F. Exploiting the spontaneous potential of the electrodes used in capacitive mixing technique for extraction of salinity-difference energy. Energy Environ. Sci. 2012, 5, 16934-16938.

18. Brogioli, D. Extracting renewable energy from a salinity difference using a capacitor. Phys. Rev. Lett. 2009, 103, 058501.

19. Sales, B.B.; Saakes, M.; Post, J.; Buisman, C.J.N.; Biesheuvel, P.M.; Hamelers, H.V.M. Direct power production from a water salinity difference in a membrane-modified supercapacitor flow cell. Environ. Sci. Technol. 2010, 44, 5661-5665.

20. La Mantia, F.; Pasta, M.; Deshazer, H.D.; Logan, B.E.; Cui, Y. Batteries for efficient energy extraction from a water salinity difference. Nano Lett. 2011, 11, 1810-1813.

21. Oren, Y. Capacitive deionization (CDI) for desalination and water treatment- past, present and future (a review). Desalination 2008, 228, 10-29. 
22. Biesheuvel, P.M. Thermodynamic cycle analysis for capacitive deionization. J. Colloid Interface Sci. 2009, 332, 258-264.

23. Lee, J.B.; Park, K.K.; Eum, H.M.; Lee, C.W. Desalination of a thermal power plant wastewater by membrane capacitive deionization. Desalination 2006, 196, 125-134.

24. Li, H.; Gao, Y.; Pan, L.; Zhang, Y.; Chen, Y.; Sun, Z. Electrosorptive desalination by carbon nanotubes and nanofibres electrodes and ion-exchange membranes. Water Res. 2008, 42, 4923-4928.

25. Pasta, M.; Wessells, C.D.; Cui, Y.; La Mantia. A desalination battery. Nano Lett. 2012, 12, 839-843.

26. Rica, R.A.; Ziano, R.; Salerno, D.; Mantegazza, F.; Brogioli, D. Thermodynamic relation between voltage-concentration dependence and salt adsorption in electrochemical cells. Phys. Rev. Lett. 2012, 109, 156103.

27. Van Roij, R. Electrostatics of Soft and Disordered Matter. In Proceedings of the CECAM Workshop "New challenges in Electrostatics of Soft and Disordered Matter", Toulouse, France, 7-10 May 2012; Pan Stanford Publishing: Singapore, September 2013; chapter Statistical thermodynamics of supercapacitors and blue engines, In press.

28. Lyklema, J. Fundamentals of Interface and Colloid Science, Volume 2; Academic Press: New York, NY, USA, 1995.

29. Lakshminarayanaiah, N. Transport phenomena in artificial membranes. Chem. Rev. 1965, 65, 491-565.

30. Rica, R.A.; Bazant, M.Z. Electrodiffusiophoresis: Particle motion in electrolytes under direct current. Phys. Fluids 2010, 22, 112109.

31. Brogioli, D.; Zhao, R.; Biesheuvel, P.M. A prototype cell for extracting energy from a water salinity difference by means of double layer expansion in nanoporous carbon electrodes. Energy Environ. Sci. 2011, 4, 772-777.

32. Liu, F.; Schaetzle, O.; Sales, B.B.; Saakes, M.; Buisman, C.J.N.; Hamelers, H.V.M. Effect of additional charging and current density on the performance of Capacitive energy extraction based on Donnan Potential. Energy Environ. Sci. 2012, 5, 8642-8650.

33. Burheim, O.; Sales, B.; Schaetzle, O.; Liu, F.; Hamelers, H.V.M. Auto generative capacitive mixing for power conversion of sea and river water by the use of membranes. J. Energy Resour. Technol. 2012, doi:10.1115/1.4007717.

34. Bazant, M.Z.; Thornton, K.; Ajdari, A. Diffuse-charge dynamics in electrochemical systems. Phys. Rev. E: Stat. Nonlinear Soft Matter Phys. 2004, 70, 021506.

35. Callen, H.B. Thermodynamics and an Introduction to Thermostatistics; John Wiley and Sons: Hoboken, NJ, USA, 1985.

36. Biesheuvel, P.M.; Bazant, M.Z. Nonlinear dynamics of capacitive charging and desalination by porous electrodes. Phys. Rev. E Stat. Nonlinear Soft Matter Phys. 2010, 81, 031502.

37. Biesheuvel, P.M.; Fu, Y.; Bazant, M.Z. Diffuse charge and Faradaic reactions in porous electrodes. Phys. Rev. E Stat. Nonlinear Soft Matter Phys. 2011, 83, 061507.

38. Schmuck, M. Modeling and deriving porous media Stokes-Poisson-Nernst-Planck equations by a Multi-Scale approach. Commun. Math. Sci. 2011, 9, 685-710. 
39. Mani, A.; Bazant, M.Z. Deionization shocks in microstructures. Phys. Rev. E 2011, 84, 061504.

40. Schmuck, M. First error bounds for the porous media approximation of the Poisson-Nernst-Planck equations. Z. Angew. Math. Mech. 2012, 92, 304-319.

41. Ferguson, T.R.; Bazant, M.Z. Nonequilibrium thermodynamics of porous electrodes. J. Electrochem. Soc. 2012, 159, A1967-A1985.

42. Schmuck, M.; Berg, P. Homogenization of a catalyst layer model for periodically distributed pore geometries in PEM fuel cells. Math. Phys. 2012, arXiv:1204.6698.

43. Biesheuvel, P.; Fu, Y.; Bazant, M. Electrochemistry and capacitive charging of porous electrodes in asymmetric multicomponent electrolytes. Russ. J. Electrochem. 2012, 48, 580-592.

44. Rica, R.A.; Brogioli, D.; Ziano, R.; Salerno, D.; Mantegazza, F. Ions transport and adsorption mechanisms in porous electrodes during capacitive-mixing double layer expansion (CDLE). J. Phys. Chem. C 2012, 116, 16934-16938.

45. Rica, R.A.; Ziano, R.; Salerno, D.; Mantegazza, F.; Bazant, M.Z.; Brogioli, D. Electro-diffusion of ions in porous electrodes for capacitive extraction of renewable energy from salinity differences. Electrochim. Acta 2013, 92, 304-314.

46. Hess, K.; Epting, W.; Litster, S. Spatially resolved, in situ potential measurements through porous electrodes as applied to fuel cells. Anal. Chem. 2011, 83, 9492-9498.

47. Hess, K.C.; Whitacre, J.F.; Litster, S. In situ measurements of potential, current and charging current across an EDL capacitance anode for an aqueous sodium hybrid battery. J. Electrochem. Soc. 2012, 159, A1351-A1359.

48. Sales, B.B.; Burheim, O.S.; Liu, F.; Schaetzle, O.; Buisman, C.J.N.; Hamelers, H.V.M. Impact of wire geometry in energy extraction from salinity differences using capacitive technology. Environ. Sci. Technol. 2012, 46, 12203-12208.

49. Burheim, O.S.; Liu, F.; Sales, B.B.; Schaetzle, O.; Buisman, C.J.N.; Hamelers, H.V.M. Faster time response by the use of wire electrodes in capacitive salinity gradient energy systems. J. Phys. Chem. C 2012, 116, 19203-19210.

50. Wang, H.; Pilon, L. Mesoscale modeling of electric double layer capacitors with three-dimensional ordered structures. J. Power Sources 2013, 221, 252-260.

51. Bazant, M.Z.; Kilic, M.S.; Storey, B.D.; Ajdari, A. Towards an understanding of induced-charge electrokinetics at large applied voltages in concentrated solutions. Adv. Colloid Interface Sci. 2009, 152, 48-88.

52. Jiménez, M.L.; Fernández, M.M.; Ahualli, S.; Iglesias, G.; Delgado, A.V. Predictions of the maximum energy extracted from salinity exchange inside porous electrodes. J. Colloid Interface Sci. 2013, doi:10.1016/j.jcis.2013.03.068.

53. Delgado, A.; González-Caballero, F.; Hunter, R.; Koopal, L.; Lyklema, J. Measurement and interpretation of electrokinetic phenomena. J. Colloid Interface Sci. 2007, 309, 194-224.

54. Carrique, F.; Arroyo, F.J.; Delgado, A.V. Electrokinetics of concentrated suspensions of spherical colloidal particles with surface conductance, arbitrary zeta potential, and double-layer thickness in static electric fields. J. Colloid Interface Sci. 2002, 252, 126-137.

55. Chmiola, J.; Yushin, G.; Gogotsi, Y.; Portet, C.; Simon, P.; Taberna, P.L. Anomalous increase in carbon capacitance at pore sizes less than 1 nanometer. Science 2006, 313, 1760-1763. 
56. Porada, S.; Weinstein, L.; Dash, R.; van der Wal, A.; Bryjak, M.; Gogotsi, Y.; Biesheuvel, P. Water desalination using vapacitive deionization with microporous carbon electrodes. ACS Appl. Mater. Interfaces 2012, 4, 1194-1199.

57. Conway, B.E. Transition from "supercapacitor" to "battery" behavior in electrochemical energy storage. J. Electrochem. Soc. 1991, 138, 1539-1548.

58. Lee, J.H.; Bae, W.S.; Choi, J.H. Electrode reactions and adsorption/desorption performance related to the applied potential in a capacitive deionization process. Desalination 2010, 258, 159-163.

59. Sales, B.B.; Liu, F.; Schaetzle, O.; Buisman, C.J.; Hamelers, H.V. Electrochemical characterization of a supercapacitor flow cell for power production from salinity gradients. Electrochim. Acta 2012, 86, 298-304.

60. Andersen, M.B.; van Soestbergen, M.; Mani, A.; Bruus, H.; Biesheuvel, P.M.; Bazant, M.Z. Current-induced membrane discharge. Phys. Rev. Lett. 2012, 109, 108301.

61. Porada, S.; Sales, B.B.; Hamelers, H.V.M.; Biesheuvel, P.M. Water desalination with wires. J. Phys. Chem. Lett. 2012, 3, 1613-1618.

62. Turek, M.; Bandura, B.; Dydo, P. Power production from coal-mine brine utilizing reversed electrodialysis. Desalination 2008, 221, 462-466.

63. Cipollina, A.; Misseri, A.; D’Alí Staiti, G.; Galia, A.; Micale, G.; Scialdone, O. Integrated production of fresh water, sea salt and magnesium from sea water. Desalin. Water Treat. 2012, 49, 390-403.

64. Vermaas, D.; Bajracharya, S.; Bastos, B.; Saakes, M.; Hamelers, H.V.; Nijmeijer, K. Clean energy generation using capacitive electrodes in reverse electrodialysis. Energy Environ. Sci. 2013, 6, 643-651.

(c) 2013 by the authors; licensee MDPI, Basel, Switzerland. This article is an open access article distributed under the terms and conditions of the Creative Commons Attribution license (http://creativecommons.org/licenses/by/3.0/). 